\title{
The Short Baseline Neutrino Program at Fermilab
}

\section{Joel Mousseau*}

University of Michigan

E-mail: joelameumich.edu

The Fermilab Short Baseline Neutrino program has been designed to answer several outstanding questions in the field of neutrino oscillation physics. From the LSND result to the MiniBooNE low energy excess, numerous anomalous results can be tested with the Short Baseline Program's configuration of three neutrino detectors. Each detector employs liquid argon time projection chambers to enable superior electron gamma separation needed to untangle the various questions surrounding neutrino physics. MicroBooNE is currently taking data, and has already begun the process of reconstructing and analyzing events in liquid argon TPCs. While MicroBooNE will be able to determine the nature of MniBooNE's low energy excess, it will be up to the combination of Icarus and the Short Baseline Near Detector to definitively prove or disprove the existence of large $\Delta m^{2}$ oscillations.

XVII International Workshop on Neutrino Telescopes

13-17 March 2017

Venezia, Italy

${ }^{*}$ Speaker. 


\section{Introduction}

Beginning in the 1990s, evidence began to mount for the existence of a fourth species of neutrino from a variety of sources. Due to measurements from LEP, which constrain the number of neutrinos which can interact weakly based on the width of the $\mathrm{Z}$ decays, this hypothetical fourth neutrino is "sterile," or does not feel the weak nuclear force. In all cases, this neutrino has not been directly observed but instead inferred from the appearance of electron neutrinos in a beam of primarily muon neutrinos.

While the appearance of electron neutrinos from a beam of muon neutrinos has been observed in many experiments, in each case the distance between the muon neutrino source and detector has been on the order of $\mathrm{km}$. The LSND experiment, located at Los Alamos National Laboratory, observed a $3.8 \sigma$ excess of electron neutrinos over a baseline of several meters [1]. Combined with the muon neutrino energy of a few $\mathrm{MeV}$, this leads to a $\Delta M^{2}$ between the muon and fourth neutrino on the order of $1 \mathrm{eV}^{2}$. This contrasts with other observed mass mixing parameters, which are typically on the order of $10^{-6} \mathrm{eV}^{2}$ (see Figure 1).

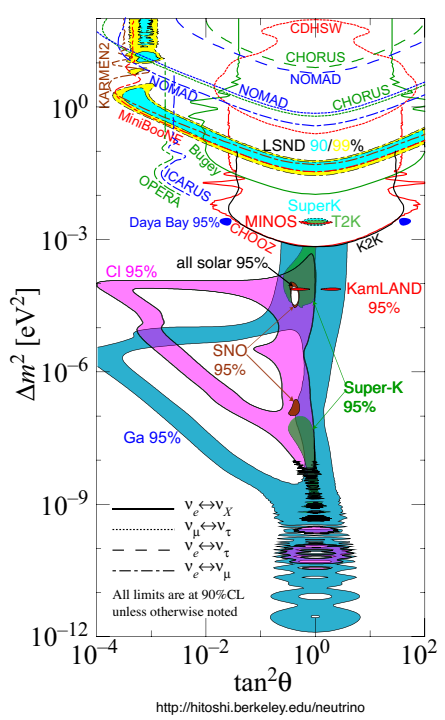

Figure 1: Measured $\Delta m^{2}$ and $\tan ^{2}(\theta)$ space of $v_{\mu}$ to $v_{e}$ oscillations. Included regions are shown as colored areas, and excluded regions are given as regions enclosed by solid lines. Note the LSND signal located in the top left region at a $\Delta m^{2} \approx 1 \mathrm{eV}^{2}$, which is several orders of magnitude larger than the SNO result. Figure is from Ref. [2].

The MiniBooNE experiment, which began taking data in 2002, was designed to confirm or refute the observed excess of electron neutrinos seen in LSND. MiniBooNE consists of a spherical tank filled with 800 tons of mineral oil $\left(\mathrm{CH}_{2}\right)$. Both electron and muon neutrinos were observed via the charged current quasi-elastic $(\mathrm{CCQE})$ process:

$$
v_{\mu}+n \rightarrow \mu^{-}+p^{+}, v_{e}+n \rightarrow e^{-}+p^{+} .
$$

These interactions are observed in MiniBooNE using PMTs along the inner wall of the tank. MiniBooNE is primarily a Cherenkov detector, and discriminates between muons and electrons based 
on the presence or absence of Cherenkov rings observed by the PMTs.

While MiniBooNE did not confirm the LSND result, it did observe an excess of events in both electron neutrino and electron anti-neutrino in the energy range of 200 to $475 \mathrm{MeV}$. This is illustrated in Figure 2, which shows an excess of data over the expected backgrounds. It should be noted that the dominate background at low energies in MiniBooNE is the production of $\pi^{0} \mathrm{~s}$ which decay strongly to two photons. As MiniBooNE is not able to distinguish electrons and photons precisely, a potential origin of this excess in mis-identified and mis-modeled $\pi^{0} \mathrm{~s}$ created by muon neutrinos.

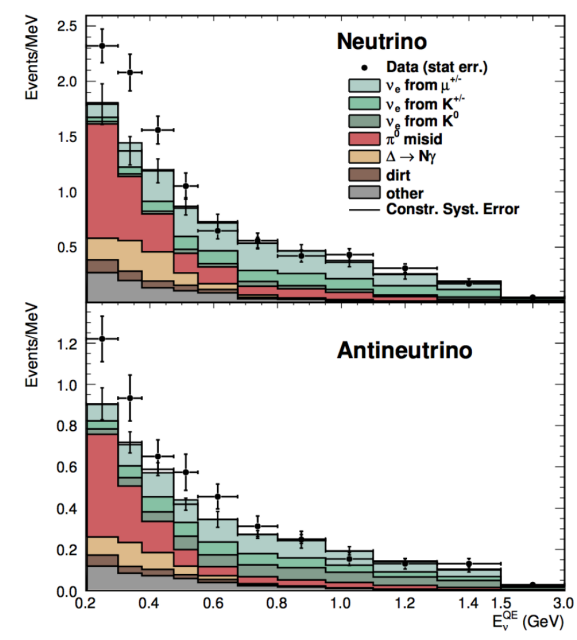

Figure 2: Number of neutrino electron like events observed (points) and the expected backgrounds broken up by simulated channel as a function of neutrino energy $E_{v}$. The error bars on the data are statistical, and the error bars on the simulation are systematic uncertatinties. The excess of data events in neutrino mode (top) and anti-neutrino mode (bottom) in the region of 200 to $475 \mathrm{MeV}$ has a significance of about $3 \sigma$. Figures are from Ref. [3] and [4].

\section{Electron/Photon Separation with Liquid Argon Time Projection Chambers}

Liquid Argon Time Projection Chambers (LAr TPCs) are a promising technology for current and future neutrino oscillation experiments. Charged particles resulting from neutrino interactions ionize the neutral argon ions, and the resulting electrons drift in and electric field to readout wires. Discrimination between electrons and photons is achieved through measuring the $\frac{d E}{d x}$ of tracks within the argon. Photons deposit roughly a factor of 2 more energy per unit lengths than electrons due to pair production. This has been measured by the ArgoNeut experiment [5], and is described in Figure 3.

The drift time of electrons in the $27 \mathrm{kV} / \mathrm{m}$ electric fields in argon is long (several ms) compared to the width of the neutrino beam spill (several $\mu \mathrm{s}$ ). As a result, photo multiplier tubes (PMTs) are used to detect the scintillation light of charged particles traveling through argon. This light arrives very promptly (within several ns), and allows TPCs to align tracks with a beam spill, as well as trigger the TPC based on a threshold of scintillation light. 


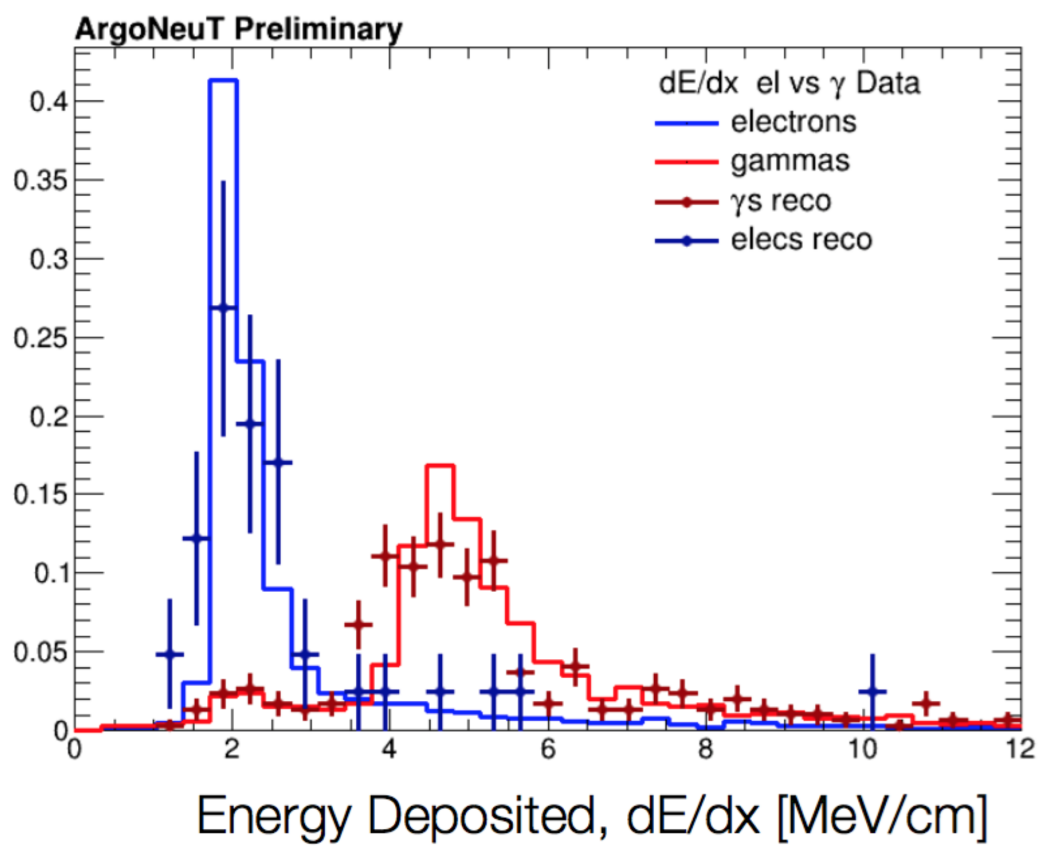

Figure 3: Energy deposited per unit lenght in argon for identified electrons (blue) and identified photons (red) in the ArgoNeuT detector. The solid lines are the simulation, and the points are data. Figure is from Ref. [5].

\section{Introduction to the Short Baseline Program}

In an effort to deduce the electron or photon nature of the MiniBooNE excess, as well as fully understand the origin of the LSND excess, Fermi National Accelerator Laboratory (Fermilab) is in the process of building and commissioning the Short Baseline Neutrino Program. This program will consist of three detectors each employing LAr TPC technology, all exposed to the booster neutrino beam (BNB) at Fermilab. The usage of three detectors enables a near and far detector comparison of neutrino interaction rates, which in turn leads to a more precise measurement of oscillation parameters. Each detector, along with its' distance from the neutrino source and total liquid argon mass is presented in Figure 4.

Of the three detectors that make up the short baseline program, Icarus is the largest, and enables $5 \sigma$ sensitivity to the LSND excess if it is present. Icarus has been recently delivered to Fermilab from CERN, and is awaiting installation. The detector closest to the beam source, SBND (Short Baseline Near Detector), is currently under construction. Finally MicroBooNE is currently operating and taking data using the neutrino beam.

While all three of the Short Baseline detectors are important for an oscillation search, MicroBooNE and SBND in particular are important for studying neutrino cross-sections and understanding the technology of LAr TPCs. This will become especially true as future, larger LAr TPCs begin operation and constructions such as the DUNE far detector. 


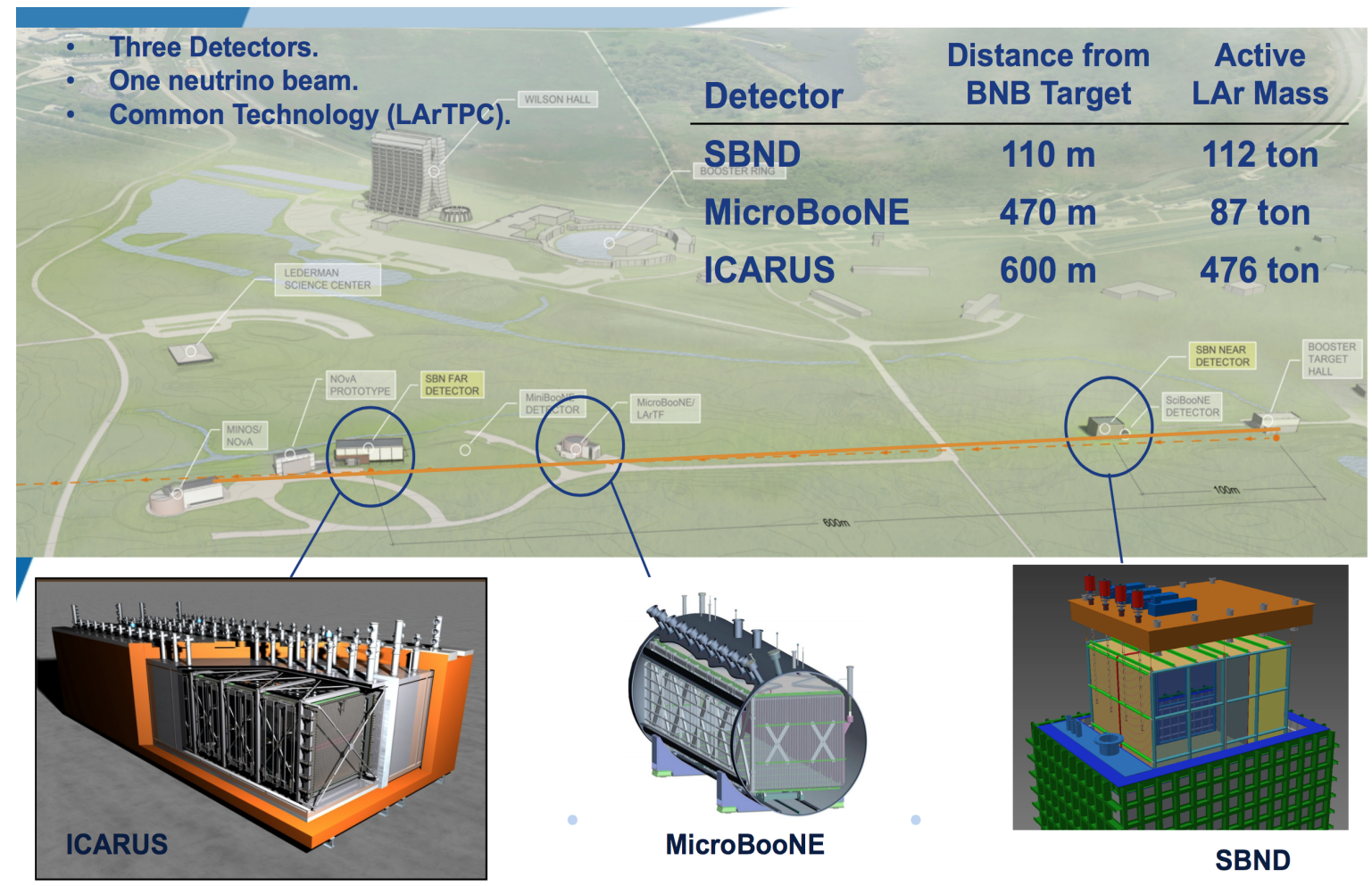

Figure 4: Outline of the short baseline neutrino program at Fermialb. The neutrino beam is provided by the booster neutrino beam, and enters via the right hand side of the page. All three detectors, Icarus, MicroBooNE and SBND are liquid argon TPCs of varying size. Icarus has recently arrived at Fermilab, and SBND is currently under construction. MicroBooNE is currently running and is collecting data from the BNB and NuMI (not pictured) neutrino beams. The MicroBooNE TPC has dimenstions of $2.5 \times 2.3 \times 10.4$ meters. 


\section{The Short Baseline Near Detector (SBND)}

Being the detector nearest to the neutrino source, SBND will be responsible for measuring the un-oscillated neutrino flux. While the BNB is $\approx 98 \% v_{\mu}$, there is a few percent component of $v_{e}$ s intrinsic to the beam. As a result, any single detector oscillation experiment will observe some number of $v_{e}$ s not due to oscillations. Using a near detector such as SBND allows any excess of electron neutrino like events in the far detector (ICARUS) to be directly interpreted as an oscillation signal. This technique greatly improves the SBN's sensitivity to an LSND like oscillation signal as shown in Figure 5.

SBND is currently under construction, with the assemblies for the TPC anodes and cathode underway. The cryostat is scheduled to be installed in 2018, and data taking is slated to begin in early 2019. SBND's proximity to the neutrino source as well as large mass will enable it to make very precise neutrino cross-section measurements. SBND expects nearly 5 million CC $v_{\mu}$ events over a 3 year run, and nearly 40 thousand $\mathrm{CC} v_{e}$ events from $v_{e}$ s intrinsic to the BNB over the same period.

In addition to being a laboratory for studying neutrino-argon cross-sections, SBND will also be a test bed for future liquid argon research and development projects. One project being pursued by SBND for the collection of scintillation light is the usage of wavelength shifting coated acrylic light bars. Detecting scintillation light from liquid argon is essential for LAr TPCs to reduce cosmic ray backgrounds by determining the timing of tracks relative to the arrival of the neutrino beam. The SBND light bars are supported vertically within the SBND anode plane assemblies, and work in tandem with the PMT system in SBND. These light bars use silicon photomultipliers (SiPMs) to detect light from the bars. The bar and SiPM combination SBND is investigating is similar to the design considered by the DUNE and proto-DUNE experiments.

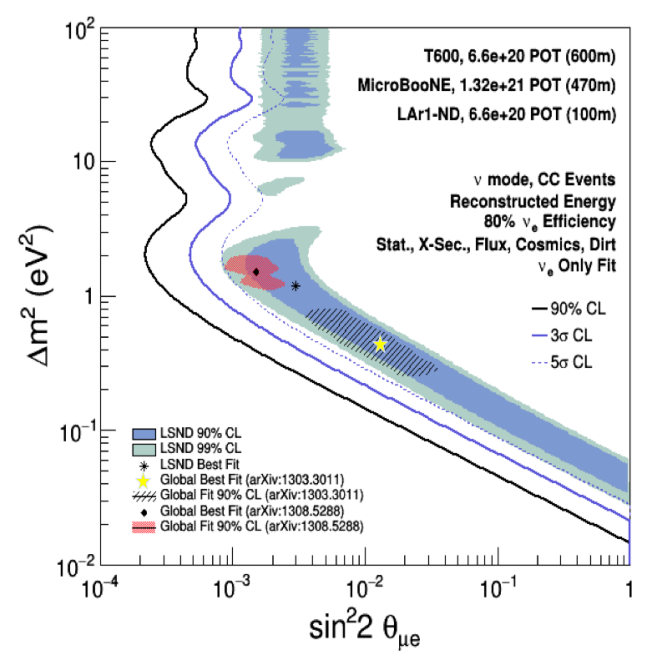

Figure 5: SBN sensitivity overlyaed on top of the observed LSND oscillation space, as well as several global best fit points. The combined three SBN detectors, LAr1-ND (SBND), MicroBooNE and T600 (ICARUS) allow $5 \sigma$ coverage of the entire LSND space, as well as the three best fit points. Figure is from Ref [6]. 


\section{The MicroBooNE Experiment}

\subsection{Event Reconstruction in MicroBooNE}

MicroBooNE has currently take approximately $6 \times 10^{20}$ protons on target (POT) of neutrino data from the BNB beam. A small subset of that data, $5 \times 10^{19}$ POT have been used to preform various detector and physics studies. Reconstruction begins in MicroBooNE with a deconvolution stage, which factors out the response time of the wires and readout electronics from the raw wire signal. Deconvolved signals are sorted into discrete detector hits when a wire signal is found to be above baseline. This procedure is prone to errors if the wire signal is especially noisy, which can create a number of spurious noise hits. MicroBooNE's software contains a noise finding algorithm which tags these hits and removes them from consideration of downstream reconstitution.

MicroBooNE's downstream reconstruction sorts two dimensional hits into spatially correlated clusters. Clusters are then classified by the algorithm into shower or track like depending on the proximity of neighboring clusters. Track like clusters are stitched across three dimensions to form 3D tracks. Shower like clusters are likewise stitched to form 3D showers. Showers and tracks form the building blocks of more abstract objects such as particles and vertices. Vertices contained within the TPC are marked as potential neutrino vertices. All of the track, shower and neutrino reconstruction are preformed as part of the Pandora package, documented in Ref [7].

Once all tracks have been constructed in the TPC, a second stage of reconstruction matches TPC tracks to depositions of light in the MicroBooNE PMTs. These depositions of light are called flashes, and matching tracks to them in called flash matching. Flash matching allows the timing information of the track to be measured at the $10 \mathrm{~ns}$ level. In this way, only neutrino vertices which are detected within the neutrino beam are considered for analysis. This procedure dramatically decreases the cosmic ray contamination. Preliminary data and MC comparisons of track matching variables, including number of photo-electrons per flash are in Figure 6. MicroBooNE has made several improvements to the optical simulation, and believes this agreement will improve in the near future.
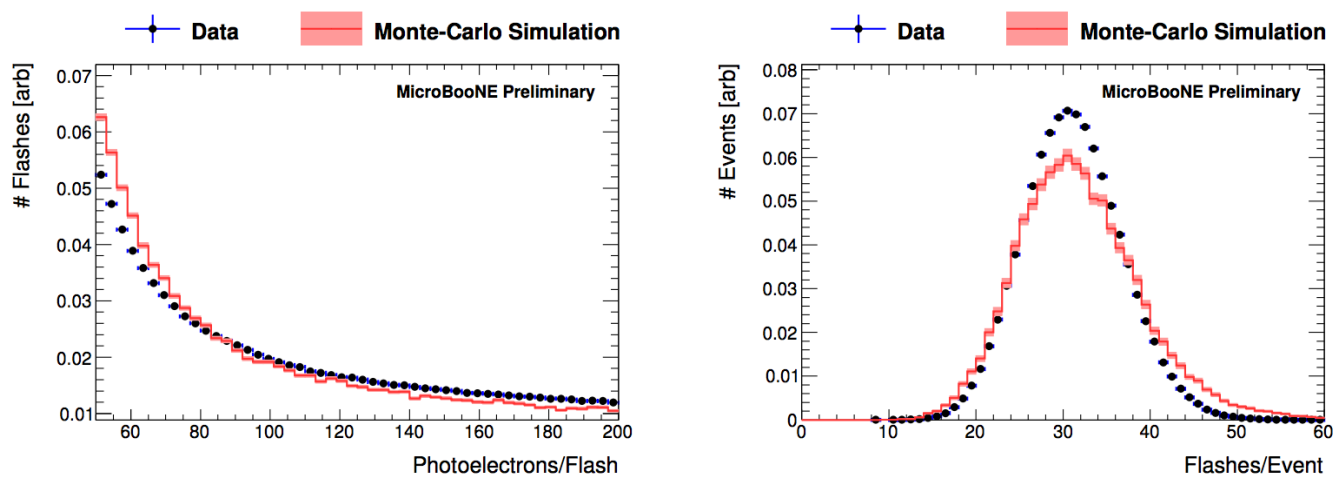

Figure 6: Data and MC comparions of the number of photoelectrons per flash (left) as well as the number of flashes per event (right). Figure is from Ref [8]. 


\subsection{Understanding the Space Charge Effect}

Cosmic rays continuously ionize argon atoms within surface TPCs. These positively charged ionize drift toward the negatively charged cathode of the TPC, where they form a cloud of positive charge which deflects drifting electrons and distorts the drift field. This space charge effect must be calibrated out of the reconstruction in order to obtain accurate tracking information. MicroBooNE currently models the space charge effect in simulation. The deflection due to simulated space charge has been tested against data and shown to agree for positive deflections, however there is some disagreement for negative deflections. This is displayed in Figure 7.

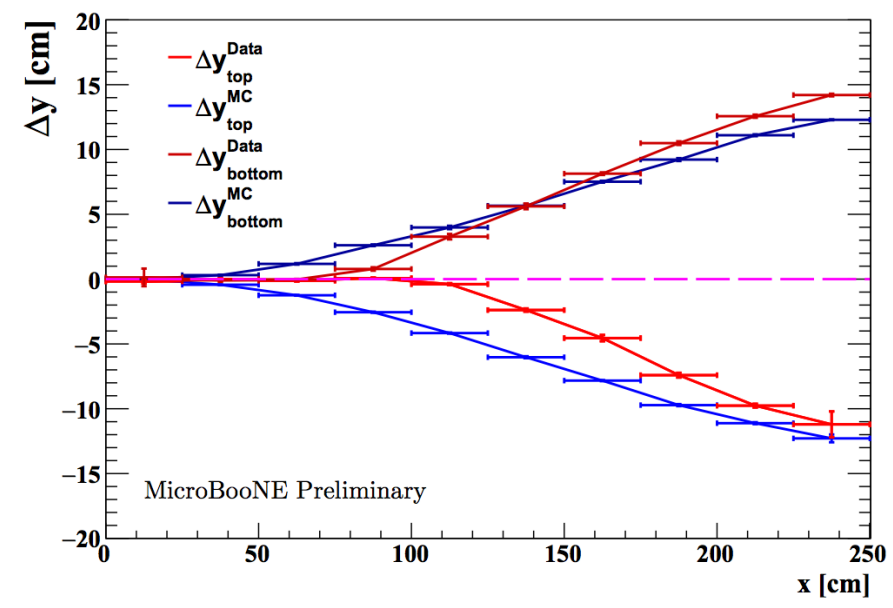

Figure 7: Deflections in the $y$ (vertical) directions of tracks as a funciton of $x$ (along the drift direction) due to the space charge effect. Note that the defelctions grow as the tracks approach the cahotde, $x \rightarrow 250 \mathrm{~cm}$. The simulated effect is shown to agree well with data for both positive (upward) deflections. Figure is from Ref [9].

\subsection{Michel Electron Tagging}

Michel electron tagging in MicroBooNE begins once the tracking algorithm has completed. Long cosmogenic stopping muons are identified by locating the Bragg peak at the end of long minimum-ionizing tracks. The decay electron from the stopped muon continues to propagate through the detector as a continuous track. As it's possible the electron can produce radiative photons which produce hard bremsstrahlung electrons in the detector, the reconstruction extends the electron track past it's end point to add in the extra energy. This procedure dramatically improves the energy resolution of the algorithm. The final distribution of data and simulated Michel electrons is presented in Figure 8. Reconstruction of the Michel spectrum is important for TPCs to validate their tracking and reconstruction chain. In addition, the electron energy spectrum serves to calibrate the absolute energy scale of the detector, which is essential in fully reconstructing neutrino energy from beam events. 


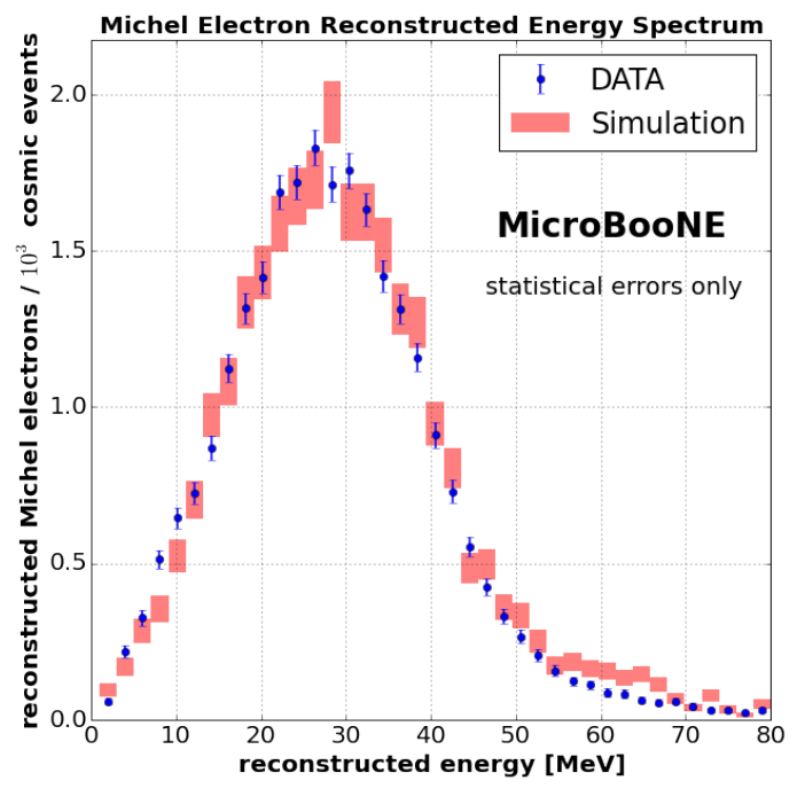

Figure 8: Energy spectrum of michel electrons after accounting for energy from radiative electrons. The statistical uncertainty is drawn as lines around the blue data points. Figure is from Ref. [10].

\subsection{Charged Current Inclusive Analysis in MicroBooNE}

Charged current inclusive incorporates all neutrino-nucleon interactions with a muon in the final state. Cutting on events with at least one muon detected by the TPC creates a sample of almost completely pure $v_{\mu}$. Beyond the normal flash matching discussed in Section 5.1, a number of other cuts are imposed to reduce the contamination of cosmic ray muons. These cuts create two sub-samples of inclusive events named "Selection 1" and "Selection 2." The largest differences between Selection 1 and Selection 2 are Selection one demands the muon track be fully contained within the fiducial TPC volume, and Selection 2 allows the muon to exit if additional tracks are present. In addition, Selection 1 requires the muon track to be at least $75 \mathrm{~cm}$ long, where Selection 2 makes on such requirement.

The track length and track angle with respect to the neutrino beam are measured in inclusive events. These are compared to the simulation after an area normalization in Figure . There is in general good agreement between the data and simulation, after remaining cosmic backgrounds are subtracted from distributions of off-beam data. The remaining backgrounds consist of neutral current events where a muon was mistakenly tagged, and cosmic events which overlapped with a neutrino event.

Future plans for MicroBooNE include using this charge current inclusive selection process to produce a cross-section in terms of neutrino energy and track multiplicity. These cross-section results will enable comparisons and provide tuning parameters for the GENIE event generator, which is used by MicroBooNE and many other neutrino experiments. 

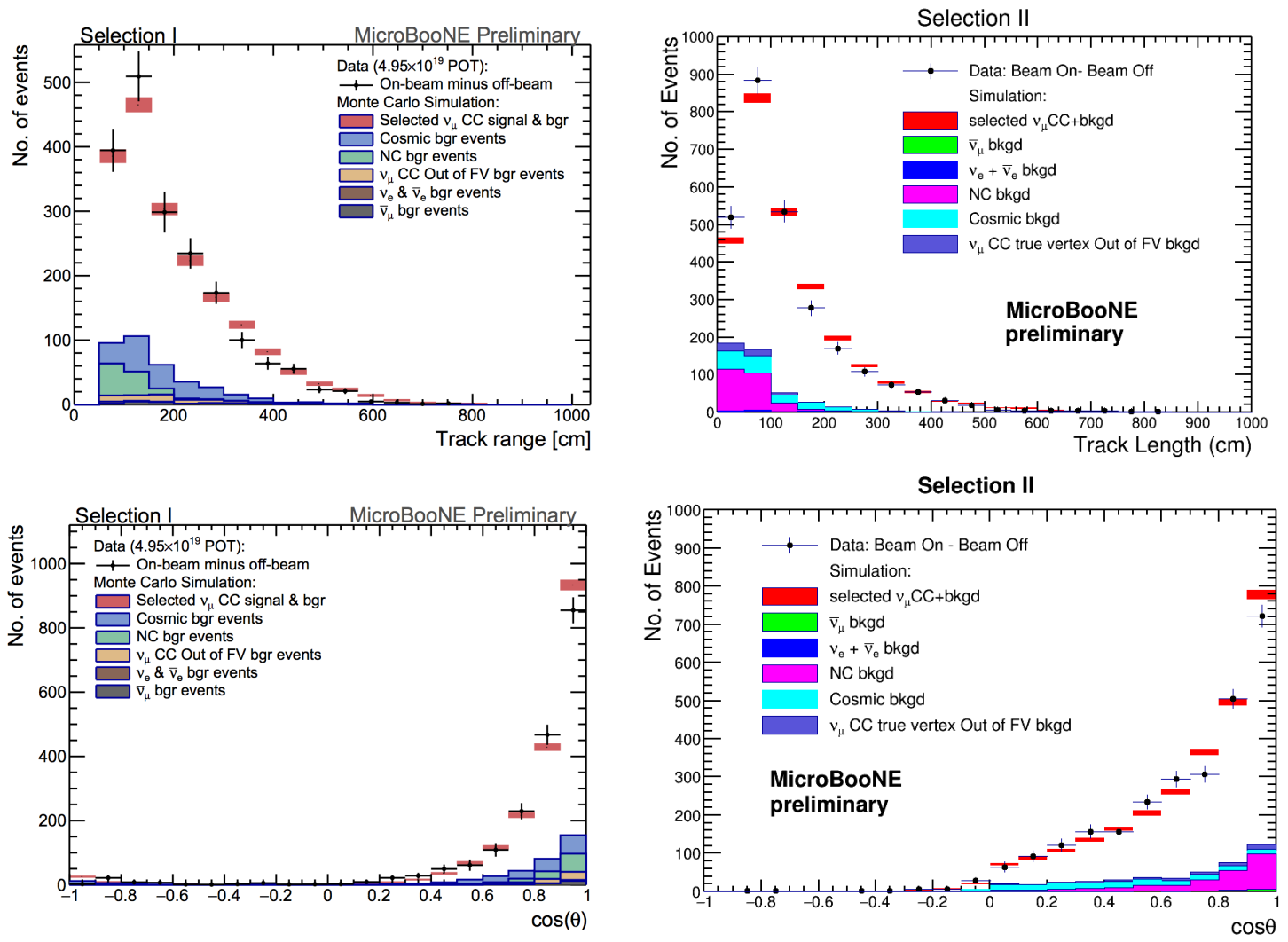

Figure 9: Top: track length in cm of daughter muons from charged-current inclusive events from Selection 1 (right) and Selection 2 (left). Bottom angle with respect to the beam of muons for Selection 1 (right) and Selection 2 (left). Distributions are drawn for both data and simulation. In all cases, the backgrounds are broken down into their various components. Figures are form Ref [11]. 


\section{Conclusions}

The Short Baseline program at Fermilab is a promising avenue for deducing the origin of the MiniBooNE and LSND excesses. Icarus, the planned far detector, has already arrived at Fermilab and is in the process of installation. SBND, the near detector, is in the process of construction. In addition, SBND will be used as a test bed for promising new light detection technology. MicroBooNE is currently operating, and has already made several important contributions to LAr TPC physics in the form of Michel electron tagging and characterization of the space charge effect. The inclusive selections developed by MicroBooNE will be used in the near future to make detailed inclusive cross-section measurements vital to the further development of GENIE. With three experiments working together, the Short Baseline program is poised to be fertile ground for discovery.

\section{References}

[1] A. Aguilar-Arevalo et al. [LSND Collaboration], Phys. Rev. D 64, 112007 (2001) doi:10.1103/PhysRevD.64.112007 [hep-ex/0104049].

[2] K. A. Olive et al. [Particle Data Group], Chin. Phys. C, 38, 090001 (2014) and 2015 update.

[3] A. A. Aguilar-Arevalo et al. [MiniBooNE Collaboration], Phys. Rev. Lett. 102, 101802 (2009) doi:10.1103/PhysRevLett.102.101802 [arXiv:0812.2243 [hep-ex]].

[4] A. A. Aguilar-Arevalo et al. [MiniBooNE Collaboration], Phys. Rev. Lett. 105, 181801 (2010) doi:10.1103/PhysRevLett.105.181801 [arXiv:1007.1150 [hep-ex]].

[5] R. Acciarri et al. [ArgoNeuT Collaboration], Phys. Rev. D 95, no. 7, 072005 (2017) [Phys. Rev. D 95, 072005 (2017)] doi:10.1103/PhysRevD.95.072005 [arXiv:1610.04102 [hep-ex]].

[6] M. Antonello et al. [MicroBooNE and LAr1-ND and ICARUS-WA104 Collaborations], arXiv:1503.01520 [physics.ins-det].

[7] R. Acciarri et al. [MicroBooNE Collaboration], [arXiv:1708.03135 [hep-ex]].

[8] MicroBooNE Collaboration MICROBOONE-NOTE-1014-PUB. https://www-microboone.fnal.gov/publications/publicnotes/MICROBOONE-NOTE-1014-PUB.pdf

[9] MicroBooNE Collaboration MICROBOONE-NOTE-1017-PUB. https://www-microboone.fnal.gov/publications/publicnotes/MICROBOONE-NOTE-1017-PUB.pdf

[10] R. Acciarri et al. [MicroBooNE Collaboration], [arXiv:1704.02927 [physics.ins-det]].

[11] MicroBooNE Collaboration MICROBOONE-NOTE-1010-PUB. https://www-microboone.fnal.gov/publications/publicnotes/MICROBOONE-NOTE-1010-PUB.pdf 